Service social

\title{
Autonomie et expérience de la dépendance chez les personnes âgées ayant subi un traumatisme craniocérébral
}

\author{
Éric Gagnon, Manon Voyer, Paule Terreau, Nadine Lacroix, Geneviève \\ Fournier St-Amand et Marie-Christine Ouellet
}

Volume 62, numéro 2, 2016

URI : https://id.erudit.org/iderudit/1038576ar

DOI : https://doi.org/10.7202/1038576ar

Aller au sommaire du numéro

Éditeur(s)

École de travail social et de criminologie de l’Université Laval

ISSN

1708-1734 (numérique)

Découvrir la revue

Citer cet article

Gagnon, É., Voyer, M., Terreau, P., Lacroix, N., Fournier St-Amand, G. \& Ouellet, M.-C. (2016). Autonomie et expérience de la dépendance chez les personnes âgées ayant subi un traumatisme craniocérébral. Service social, 62(2), 49-63. https://doi.org/10.7202/1038576ar

\section{Résumé de l'article}

Le retour à domicile des personnes âgées ayant subi un traumatisme craniocérébral pose la question de leur autonomie. La question porte autant sur leur autonomie fonctionnelle, leur capacité à réaliser les activités de la vie quotidienne, que leur autonomie décisionnelle, leur participation à la prise de décision touchant leur lieu de résidence. Le présent article examine les conditions d'exercice de cette double autonomie et l'expérience de la dépendance chez les personnes ayant subi un traumatisme craniocérébral, dans l'objectif d'améliorer l'accompagnement offert à ces personnes pendant et après la réadaptation. L'analyse repose sur des entretiens avec des personnes âgées et leurs proches. 


\title{
Autonomie et expérience de la dépendance chez les personnes âgées ayant subi un traumatisme craniocérébral
}

\author{
GAGNON, Éric \\ Professeur associé \\ Faculté des sciences sociales \\ Université Laval \\ VOYER, Manon \\ Ergothérapeute \\ IRDPQ \\ CIUSSS-CN \\ TERREAU, Paule \\ Coordonnatrice clinique \\ IRDPQ \\ CIUSSS-CN \\ LACROIX, Nadine \\ Conseillère à la recherche \\ IRDPQ \\ CIUSSS-CN \\ FOURNIER ST-AMAND, Geneviève \\ Doctorante en psychologie \\ École de psychologie \\ Université Laval \\ OUELLET, Marie-Christine \\ Professeure adjointe \\ École de psychologie \\ Université Laval
}

\section{RÉSUMÉ}

Le retour à domicile des personnes âgées ayant subi un traumatisme craniocérébral pose la question de leur autonomie. La question porte autant sur leur autonomie fonctionnelle, leur capacité à réaliser les activités de la vie quotidienne, que leur autonomie décisionnelle, leur participation à la prise de décision touchant leur lieu de résidence. Le présent article examine les conditions d'exercice de cette double autonomie et l'expérience de la dépendance chez les personnes ayant subi un traumatisme craniocérébral, dans l'objectif d'améliorer l'accompagnement offert à ces personnes pendant et après la réadaptation. L'analyse repose sur des entretiens avec des personnes âgées et leurs proches.

Mots clés : traumatisme caniocérébral, autonomie, dépendance, réadaptation 


\section{ABSTRACT}

The autonomy of elderly people returning home after suffering a traumatic brain injury is often questioned. The issue revolves around their functional autonomy, their ability to perform activities of daily life, and their decision-making autonomy, their ability to take part in decisions that affect their place of residence. This article explores the conditions for exercising this dual autonomy and the experience with dependency among the elderly who have suffered a traumatic brain injury, with the goal of improving the support provided during and after rehabilitation. The analysis is based on interviews held with elderly people and their relatives.

Keywords: traumatic brain injury, autonomy, dependency, rehabilitation 
II n'est pas un secteur d'intervention aujourd'hui, où il n'est question d'autonomie; pas une seule politique sociale, programme ou pratique qui ne vise à restaurer ou à maintenir l'autonomie fonctionnelle de l'usager, sa capacité de subvenir lui-même à ses besoins, tout en lui reconnaissant une autonomie décisionnelle, le droit de juger et de décider de ce qui est bon pour lui-même. L'autonomie de la personne est à la fois la finalité de l'intervention et l'un de ses modes opératoires dans l'évaluation de la situation et la prise de décision quant aux actions et changements à entreprendre (Gagnon, 2015). II est ainsi très souvent question de consentement, de décision partagée, ou encore d'aide à la décision.

La question de l'autonomie se pose avec une insistance toute particulière dans le cas des personnes âgées ayant subi un traumatisme craniocérébral (TCC) après la période de réadaptation. La question porte alors autant sur l'autonomie fonctionnelle de la personne, sa capacité à réaliser les activités de la vie quotidienne, que sur son autonomie décisionnelle, sa participation à la décision touchant les conditions de son retour à domicile ou un éventuelle admission dans un centre d'hébergement et de soins de longue durée. Dans quelles conditions la décision est-elle prise? Qui participe à la discussion et la décision? Comment les diverses options sont-elles présentées à la personne? Quelle aide lui estelle offerte? La réponse à ces questions est loin d'être toujours claire. Elle représente même parfois une véritable épreuve, puisqu'elle met en cause l'ensemble de la vie de la personne et son organisation quotidienne, la responsabilité de ses proches et des soignants. La prise de décision et le retour à domicile sont une source d'inquiétude et d'embarras autant pour la personne âgée et ses proches, que pour les intervenants qui les accompagnent.

Nous avons exploré ces questions autour de l'expérience que les personnes âgées ayant subi un TCC et leurs proches font du handicap, de la manière dont leur existence se réorganise et du contrôle qu'elles conservent sur leur vie. Nous avons d'abord été amenés à comprendre un peu mieux comment les personnes en font leur décision - non seulement elles prennent la parole et formulent une volonté, voire un projet, mais elles prennent également les moyens pour rendre cette volonté effective. Nous avons ensuite été conduits à examiner quelques-unes des conditions d'exercice de cette autonomie, les appuis dont les personnes ont besoin pour conserver un contrôle sur leur vie, et dont la perte signifie non seulement la dépendance, mais l'immobilité, la solitude et la mort. Par la prise en considération de ces aspects, nous espérons contribuer à l'amélioration des conditions dans lesquelles la décision se prend et de l'accompagnement offert aux personnes.

\section{Préoccupations, interrogations et méthode}

La prévalence du TCC est en augmentation chez les personnes âgées de 65 ans et plus (Flanagan, Hibbard et Gordon, 2005; Goleburn et Golden, 2001). Ces dernières présentent un plus grand risque que les gens moins âgés d'un déclin fonctionnel à la suite d'un TCC, même lorsque celui-ci est évalué comme léger : l'impact du TCC est plus important pour un même degré de sévérité, et la réadaptation plus longue pour des résultats plus modestes (LeBlanc et al., 2006; Mosenthal et al., 2004; Testa et al., 2005). L'interaction entre les effets de l'atteinte cérébrale et la combinaison de facteurs liés au vieillissement normal ou pathologique ajoute à la complexité. Chez les personnes âgées, le TCC peut causer des problèmes au plan moteur ou sensoriel (ex. paralysie, parésie, spasticité, problèmes visuels) et des déficits au niveau cognitif (ex. problèmes de mémoire, d'attention, d'organisation, de langage) (Levine et Flanagan, 2012). Sur le plan psychologique, la personne peut présenter un trouble émotionnel tel que la dépression ou l'anxiété (LeBlanc et al. 2006; Teasell et al., 2011). Même légères, 
les séquelles du TCC peuvent avoir un impact important sur l'autonomie, la sécurité et la qualité de vie de la personne âgée.

Très vite au cours de la réadaptation la question du retour et du maintien à domicile de la personne âgée ou la réorientation vers un milieu de vie alternatif est soulevée. Une décision doit alors être prise, dont les impacts sont considérables pour la personne âgée et son entourage. Si la personne retourne chez elle, les membres de son entourage vont devoir faire face à des responsabilités nouvelles ou accrues qui sont sources de fatigue, de stress et même de détresse (Degeneffe, 2001; Sinnakaruppan et Williams, 2001). La décision de recourir à l'hébergement, en revanche est souvent vécue par les proches comme une trahison envers la personne âgée (Belleau et Saint-Laurent, 2005; Ducharme, 2006). Du côté des intervenants, l'évaluation et la gestion des risques entraînent souvent chez eux un inconfort en raison de leur responsabilité en cas de problème et la crainte d'être blâmé (Atwal, Mclntyre et Wiggett, 2011); l'évaluation et la gestion des risques fait bien sûr appel à leur savoir et à leur jugement clinique mais aussi à leurs propres valeurs et à leur capacité à tolérer l'incertitude. En outre, les services publics demeurent insuffisamment développés, leur accessibilité varie d'une région à l'autre au Québec et ils ne sont pas toujours connus de la population (AQESSS, 2011). Se posent ainsi toute une série de questions touchant l'autonomie - fonctionnelle et décisionnelle - des personnes, tant les proches que les personnes ayant subi un traumatisme : leur capacité à retourner à domicile et le soutien dont elles pourront bénéficier, mais aussi leur compréhension et l'évaluation de leur situation, l'expressions de leurs désirs et de leurs volontés.

Très peu d'études ont été réalisées portant sur la prise de décision lors du retour à domicile après la période de réadaptation chez les personnes ayant subi un TCC, particulièrement auprès des aînés. Quelques-unes (Brown, Colantonio et Kim, 2012) se sont intéressées à prédire l'orientation des personnes âgées suite au congé de l'hospitalisation pour un TCC (retour à domicile, orientation en réadaptation ou orientation en établissement de soins de longue durée), mais aucune ne nous informe sur l'orientation des patients à la fin de la réadaptation, ou n'éclaire sur les conditions de la prise de décision ou l'organisation de la vie quotidienne, les difficultés qu'ils rencontrent et les enjeux que pose leur retour à domicile.

La présente étude vise précisément à éclairer les conditions entourant la prise de décision et le retour à domicile. Plus précisément, elle a pour objectif de mieux comprendre la place que prennent les aînés ayant subi un TCC et leurs proches dans la prise de décision touchant le retour à domicile et la manière dont ils s'organisent pour un retour qui correspond à leur volonté. Prenant appui sur les travaux de Paul Ricœur (2001) et de Vincent de Gaulejac (2009), nous cherchons à dégager les conditions de l'autonomie, tant décisionnelle que fonctionnelle des individus (leur capacité de dire, leur capacité de se raconter et leur capacité de faire); comment ils deviennent des sujets, c'est-à-dire comment ils acquièrent ou conservent une certaine maîtrise de leur destin; comment ils sont capables de formuler et d'exprimer un projet qui corresponde à leurs aspirations dans la continuité de ce qu'ils sont et entendent demeurer; sur quels appuis ils peuvent compter pour réaliser, au moins en partie, ce projet. Ce regard critique sur l'autonomie (ses dimensions, ses conditions d'exercice, les difficultés qu'elle soulève), permet du même coup de jeter un nouvel éclairage sur l'impact du TCC sur les projets de vie et l'identité des personnes.

L'étude a un caractère exploratoire. Elle repose sur neuf entretiens réalisés au printemps et à l'automne 2014. Cinq (5) l'on été avec des personnes âgées ayant subi un traumatisme craniocérébral - ci-après appelés « usagers » - quatre hommes et une femmes âgés entre 76 et 85 ans. Les quatre (4) autres 
entretiens ont été menées avec des proches aidantes de personnes ayant un tel traumatisme, trois conjointes et une fille ${ }^{1}$. Les personnes ayant subi un TCC (celles interrogées comme celles auxquelles les proches aidants interrogés apportent une aide) avaient reçu un diagnostic TCC léger complexe ou TCC modéré à la suite d'une chute. Elles ont été admises au Programmes des Traumatisme craniocérébraux clientèle adulte et aînée de l'Institut de réadaptation en déficience physique de Québec (IRDPQ) dans les cinq années précédant l'entrevue. La sélection s'est faite de manière à avoir une diversité de situations la plus grande possible, compte tenu de la faible taille de l'échantillon. Nous avons interrogé des hommes et des femmes, des locataires et des propriétaires, avec des personnes avec des niveaux d'atteintes cérébrale différents, des réseaux de support plus ou moins étendus, des revenus et des sources d'aide très variables. Dans tous les cas, le retour à domicile des personnes ayant subi l'accident avait fait l'objet d'une discussion au centre de réadaptation entre les usagers, les proches et les intervenants.

Le même canevas d'entretien a été utilisé pour les usagers et les proches aidants. Nous les avons interrogés sur : 1) l'histoire et la situation générale de la personnes âgée (l'accident ayant provoqué le TCC, les séquelles et les limitations en découlant), le parcours de réadaptation et son environnement domicilaire (type de résidence, réseau de soutien, contraintes); 2) la discussion entourant la prise de décision touchant le retour à domicile (les options envisagées, les avantages et inconvénients de chacune des options prises en considération, les personnes impliquées dans la prise de décision, l'option retenue et les raisons qui ont fait pencher la décision en ce sens); 3) la manière dont la personne a réagi à la prise de décision (confort, insécurité, déception, soulagement) et les raisons de cette réaction; 4) les réactions de l'entourage et des intervenants; 5) le retour au domicile (lieu et conditions d'installation, aide requise et aide reçue, les difficultés rencontrées); 6) la révision de la décision (le cas échéant), les raisons et la nouvelle installation ${ }^{2}$. Les entretiens ont par la suite été intégralement transcrits et codés. La grille de codage reprenait les grands thèmes de l'entretien, de manière à pouvoir ensuite comparer les entrevues sur ces différents thèmes, et dégager les similitudes et les différences.

Dans un premier temps nous nous arrêterons à la prise de décision et à la manière dont l'autonomie décisionnelle des personnes s'exprime fortement. Dans un second temps nous nous pencherons davantage sur l'autonomie fonctionnelle et les conditions qui permettent aux personnes de demeurer chez elles, ce qui nous conduira à l'expérience qu'elles font de la dépendance et des incapacités. Enfin, nous essayerons de dégager quelques enseignements pour l'intervention et le travail social.

\section{Désirs et volonté des usagers : la prise de décision}

Ce qui frappe en premier lieu à l'écoute des entretiens avec les personnes âgées ou leurs proches, ce sont leurs efforts pour reprendre un contrôle sur leur vie. Aucun n'est passif. Plusieurs sont même très actifs afin de trouver une solution qui leur convient.

À une exception près, les personnes interrogées - usagers ou proches aidants - désiraient un retour chez soi, dans leur appartement ou leur maison, au moment de quitter le centre de réadaptation. Pour

\footnotetext{
${ }^{1}$ Les proches aidants interrogés n'étaient pas les aidants des personnes âgées interrogées, sauf dans un cas. Les entretiens ont donc portés sur huit situations différentes. Nous ignorons l'âge des proches aidants.

${ }^{2}$ La durée des entretiens variait entre une heure et une heure et demie. Les personnes ont été sélectionnées, recrutées et interrogées par les membres de l'équipe à partir des dossiers cliniques. Le projet a été approuvé par le comité d'éthique de I'IRDPQ.
} 
elles, il n'y avait alors aucune autre option sérieusement envisageable. Leurs motivations ont un fort accent moral et identitaire. Il s'agit à tout prix de conserver leur autonomie et leur intimité, et de préserver leur lien avec leur conjoint lorsqu'elles sont en couple. Le chez-soi renvoie à leur histoire personnelle, leurs grands engagements, leur vie quotidienne, l'environnement auquel elles sont attachées, avec lequel elles sont familières et dans lequel elles trouvent une sécurité (ontologique ${ }^{3}$, sinon physique) ; le chez-soi est associé ce qui compte le plus dans leur vie et donne sens à leur existence: leurs occupations quotidiennes, leur vie de couple, la liberté de faire ce qui leur plaît, la possibilité d'être seul, de ne pas subir le contrôle ou le jugement des autres. " Moi, c'était la maison. J'ai une maison, j'ai une femme, qu'est-ce que vous voulez? [...] Je ne me suis jamais posé la question : "Où vais-je aller?" » (U2)

"C'est tellement un bon bonhomme ! Ça fait 43 ans qu'on est mariés. Puis, [...] tout le temps, ç'a été un bon gars. Alors aujourd'hui, c'est la moindre des choses que je lui rende le change, tant et aussi longtemps... Ah c'est sûr que ce n'est pas facile. Des fois je grogne, des fois je tempête, je dispute, je m'en irais... Mais ainsi va la vie, hein? [...] - Qu'est-ce qui était le plus important pour vous dans cette décision-là? - Être ensemble. C'est ça : c'est d'être ensemble. » (P3)

Des solutions intermédiaires sont parfois évoquées, quelquefois retenues, mais toujours vécues comme des compromis, un pis-aller : aller vivre chez son frère, emménager dans un petit appartement chez sa fille, limiter l'usage de sa résidence à un seul étage, emménager dans une résidence avec services. Pour plusieurs personnes, la seule option qui semble s'offrir à eux - en dehors du retour chez soi - est le centre d'hébergement et de soins de longue durée (CHSLD) ${ }^{4}$, qui est associé à la perte complète d'autonomie et d'intimité : on n'est pas tranquille nous explique une personne (U2); « il y a toujours des péteux de broue » dit un autre (U3). "Elle n'aurait jamais accepté d'être dans la même chambre qu'un autre. Maman ça fait trop longtemps qu'elle est seule dans son appartement, qu'elle est autonome, qu'elle fait ses affaires... » (P4)

Le choix semble devoir se faire entre l'autonomie et l'intimité du chez-soi d'un côté, la dépendance et la promiscuité de l'hébergement institutionnel de l'autre. Dans la discussion qu'elles disent avoir eue avec les intervenants du centre de réadaptation, le placement en centre d'hébergement semble une option toujours avancée et défendue uniquement par les intervenants. Les personnes âgées et les proches commencent par l'écarter, refusent parfois de l'envisager, et s'ils doivent s'y résoudre, dans un ou deux cas, après un séjour à la maison, c'est à contrecœur et non sans avoir tout fait pour demeurer chez eux.

Même si la collaboration est généralement bonne et que la satisfaction à l'égard des services de réadaptation reçus est grande, la différence de perspective avec les intervenants est soulignée par la personne âgée elle-même ou le proche. Rares sont ceux qui s'en remettent entièrement à l'avis des professionnels; ils ont plutôt tendance à y résister. Une majorité de répondants $(n=7)$ insistent sur l'écart, la différence de point de vue avec les intervenants. Dans le compte rendu que les usagers et les proches

\footnotetext{
${ }^{3}$ Antony Giddens (1991). La sécurité ontologique est l'inscription dans un univers qui favorise une identité stable, inscrite dans le temps (une filiation) et l'espace (une famille, un quartier, un environnement stable). Elle réduit l'anxiété face au changement, et permet d'avoir davantage confiance dans l'avenir, dans les autres et dans ses propres moyens, et ainsi d'entreprendre et de créer.

${ }^{4}$ Aucune mention n'est faite de ressources intermédiaires. D'autres options ont peut-être été proposées par les intervenants mais il est impossible de le vérifier dans le cadre de cette enquête.
} 
font des échanges avec les intervenants, ces derniers semblent insister exclusivement ou prioritairement sur les pertes, les incapacités et les risques, et favorisent l'installation dans un milieu sécuritaire après la réadaptation, alors que pour les usagers et leurs proches, la priorité est d'abord le retour chez soi, de préserver leur intimité et leur autonomie. Leur désaccord avec les intervenants ce sur point est clairement énoncé, et leur permet d'affirmer leurs préoccupations, désirs ou angoisses. «Pour vous c'était clair que vous retourniez chez vous? - Oui. - Vous n'alliez pas ailleurs? - Non. C'était chez nous »(U3).

Dans leur esprit, non seulement il est clair que ce sont eux qui ont pris la décision, mais c'est très souvent en opposition aux intervenants - quelquefois à leurs enfants - qu'ils la prennent. "On aurait dit que je mettais une barrière. [...] Je ne voulais rien entendre » (P3). Même devant la pression ou l'insistance des intervenants qui s'inquiètent pour la sécurité de l'usager et la santé du proche aidant, et cherchent à leur faire changer d'idée, ils résistent et maintiennent leur décision. « Le travailleur social me demandait si j'avais eu un lavage de cerveau ! [rires] », nous confie une proche aidante qui a ramené son mari chez elle (P2). Lorsqu'on lui demande si différentes options avaient été envisagées, une autre répond : «Oui, mais moi, je ne voulais rien entendre » (P3). Même réponse catégorique chez la plupart des usagers: "Eux autres [le centre de réadaptation] auraient voulu me garder plus longtemps. J'ai dit : "Je m'en vais chez nous, moi” » (U4).

Jamais ils ne prennent la posture de victimes ou de personnes qui se sont fait imposer des décisions, et qui ne contrôlent plus rien - même s'ils ne contrôlent pas tout. Au contraire, ils insistent sur ce qu'ils ont voulu et choisi, sur les actions qu'ils ont entreprises, les solutions qu'ils recherchent. Loin d'être passifs, ils se présentent comme des gens qui jugent, décident, agissent, malgré parfois le fardeau et l'épuisement, les inquiétudes et l'angoisse. Ainsi, lorsque les intervenants questionnent une proche aidante sur ses capacités à prendre soin de son mari, celle-ci se sent heurtée. « Ça m'a choquée. [...] L'amener à la maison avec moi, j'étais capable, j'étais forte » (P3). Même lorsque la décision les place dans une situation difficile, comme de devoir s'occuper d'une personne très peu autonome alors qu'on est soi-même âgé et fatigué, on l'assume et la fait sienne. "Ça me faisait de la peine de le laisser, donc, j'ai dit : “Je vais l'amener chez nous, je vais m'arranger" » (P1).

Lorsqu'un répondant - usager ou proche aidant - dit avoir cédé et accepté de quitter son domicile, il cherche malgré tout à reprendre le contrôle, obtient ou trouve des aménagements qui lui permettent d'accepter la décision et même de la faire sienne. Ainsi, un usager (U1) dira : «Le centre ne voulait pas que je reste seul, donc j'ai été obligé de me plier à cela. [...] J'ai accepté ce qu'ils ont à dire. J'ai fait ce qu'ils ont dit »-dans ce cas-ci aller vivre chez son frère. Mais il nous explique dans le même entretien comment il s'est organisé pour retrouver son autonomie et son intimité, reprendre en partie le contrôle sur sa vie : il a récupéré son permis de conduire et passe la journée dans sa maison, quitte à rentrer le soir chez son frère. II a ainsi repris des activités qui lui sont chères. "Quand je suis retourné chez mon frère, j'ai pris mes choses en main. » II a accepté d'aller chez son frère " pour un temps », précise-t-il, en attendant qu'il trouve le moyen de revenir chez lui, dans sa maison. Il a même refusé l'aide proposée par une travailleuse sociale « J'ai dit : "Si j'ai besoin de vous, je vous appellerai." " Comme d'autres, il refuse l'aide qui lui est offerte. L'automobile est un moyen mais aussi un symbole de l'autonomie : pouvoir se débrouiller seul, être mobile. Se déplacer en automobile lui permet d'ailleurs de reprendre ses activités de bénévolat, autre expression forte de son désir d'autonomie : " J'aime mieux donner que demander. » 
Qu'ils considèrent la décision comme étant la leur est perceptible jusque dans la façon de s'exprimer et de raconter ce qui s'est passé. Que ce soit les personnes ayant subi un TCC ou les proches aidants, ils racontent la manière dont la décision s'est prise en reconstituant les échanges avec les intervenants, ce qui les amène à parler au « Je ", à adopter la posture de celui ou celle qui prend la parole, exprime une volonté et tranche le débat : "J'ai dit à l'intervenant : "Je retourne chez moi..." » La personne est doublement le sujet de l'énoncé : elle est celle qui rapporte l'échange et celui des protagonistes de l'échange qui mène la discussion. La parole est le premier geste de reprise de contrôle. Ainsi un homme, pour qui il n'était pas question d'aller ailleurs que chez lui, nous dit l'avoir clairement signifié à son médecin : «Écoutez, Docteur, il n'est pas question que je change de maison. " À nous, il précise ensuite : « Je vais vous dire franchement, il n'y en a pas eu de discussion [...] je n'ai jamais pensé à aller ailleurs que [chez moi]. [...] La preuve c'est que je suis ici » (U2). Dans le vouloir et le pouvoir dire, un vouloir et un pouvoir faire est déjà anticipé 5 .

Reconstituer les échanges, c'est aussi faire retour sur soi, (se) dire qui l'on est, ce que l'on veut, les difficultés que l'on est prêt à assumer, ses priorités et ses choix, les raisons pour lesquelles on fait ce que l'on a décidé de faire. Une femme nous raconte cet échange avec son fils : "II me dit des fois : "Comment tu fais?" Je lui dis : "C'est mon rôle." II dit : "Ah! Tu l'aimes le père, hein?" Ben, je lui dis : "Je l'ai toujours aimé et je vais l'aimer toujours." II dit: "On est chanceux de vous avoir encore ensemble." Ce sont de petites compensations qui vont me dire merci. J'analyse un peu leur paroles... " Puis elle ajoute : « II y a des jours j'étais tannée, j'étais écœurée. Mon fils a dit : "Si je ne travaillais pas, je l'amènerais chez nous une semaine, le père." J'ai dit : "Amène-le, ça va me faire du bien." Après ça, je regrette d'avoir dit cela... mais c'est dit. Qu'est-ce que tu veux? On est des êtres humains. » (P3). Dans ces quelques phrases, elle résume sa situation, sa condition, ses difficultés, ses doutes, sa vulnérabilité, tout en assumant ses choix et leur raison profonde (l'amour pour son mari), en même temps qu'elle élargit un peu ses options, envisage graduellement de changer, trouve des arrangements provisoires pour continuer (le répit offert par le fils). Elle intègre de cette façon son passé, son présent et son proche avenir en lui donnant un sens, c'est-à-dire une cohérence et une direction à leur histoire personnelle. La personne est à la fois le sujet, l'objet et la fin de l'énoncé : c'est elle qui parle, mais se considérant à distance, de manière réflexive, elle rapporte ses propres paroles, et elle renforce du même coup sa détermination, l'image de soi, et elle prend mieux conscience de ses désirs et de sa volonté. Elle considère sa situation dans son ensemble autour d'une intention qui lui donne son sens.

L'autonomie recherchée ne se limite toutefois pas à la prise de parole et au récit qu'on en fait. Les personnes âgées comme les proches aidants prennent toutes sortes d'initiatives pour rendre leur décision possible ou se rapprocher de la solution désirée. Un usager prend ainsi l'initiative de faire davantage d'exercices de réadaptation afin de récupérer sa capacité de monter et descendre un escalier et de pouvoir ainsi retourner chez lui. «Quand j'étais à l'IRDPQ, à la personne qui s'occupe de moi j'ai dit : "Tu vas m'entraîner pour monter les marches" » (U2). À la suggestion du médecin de faire installer un ascenseur, il répond : "Vous allez travailler sur moi plutôt que me faire travailler sur ma maison. » Et après une année d'entraînement, il y parvient, en trouvant un truc pour s'aider avec sa hanche. Il fait tout pour récupérer son autonomie fonctionnelle et n'hésite pas à prendre les devants, à décider, à s'imposer. Cela ne le dispensera pas de faire des réaménagements à sa maison (ex. : installation de barres de bain, vivre sur un seul étage), mais sa capacité à monter l'escalier était

${ }^{5}$ Être sujet c'est d'abord parler à la première personne du singulier, se poser comme sujet dans la parole (Benveniste, 1974). 
une condition de son retour à la maison. « Me réhabiliter, faire des exercices, il n'y avait pas d'autres choses dans ma tête. »

Les proches aidants prennent également des initiatives. Avant la sortie de son mari, sans même en informer la travailleuse sociale qui les accompagne, une dame fait résilier son bail et trouve un appartement avec des services, mieux adapté à la condition nouvelle de son mari. Elle intervient dans l'entretien que nous réalisons avec son mari : “C'est moi qui me suis occupée de louer ici. [...] C'est moi qui me suis occupée de vendre la voiture. [...] Je ne voulais pas rester là-bas parce que je savais qu'il n'y avait pas les services voulus pour lui »(U3). Une autre ramène sa mère chez elle pour un " essai », une fin de semaine, afin de vérifier si sa mère peut retourner chez elle. Elle le fait en secret, sans le dire aux intervenants, comme elle le précise elle-même : " Je vais passer outre ce qu'ils disent » (P4). Les intervenants ont reconnu plus tard qu'elle avait raison : "Ils se sont bien aperçus que c'était vrai, qu'elle est correcte chez elle. " Une troisième ramène son mari à la maison durant les fêtes de Noël, puis décide de le garder définitivement avec elle, à l'encontre, là encore, de l'avis des intervenants qui la jugent incapable de le faire, pensent qu'elle va s'épuiser et s'inquiètent. "Tout le monde est tombé à la renverse. Ils se sont demandés : "Mais qu'est-ce que tu fais là?" » (P2). Elle devra finalement se résoudre à placer son mari en centre d'hébergement, mais non sans avoir essayé de le garder à la maison pendant quelques mois. Une quatrième, enfin, voit à la réinstallation de sa mère dans une nouvelle résidence : «On a mis la maison à vendre, mon mari dit qu'on va s'acheter un bungalow, puis il dit qu'on va s'organiser pour qu'elle [sa mère] ait son appartement à elle. [...] On va pouvoir la surveiller. Puis elle va se sentir moins isolée » (P4).

Si les personnes ne trouvent pas de réponse à toutes leurs difficultés, et que leur situation demeure souvent précaire, elles n'en sont pas moins actives. Et si elles rencontrent des difficultés, une fois de retour à la maison, elles n'expriment aucun regret et assument pleinement leur décision. Sur les huit situations étudiées, cinq personnes sont finalement retournées où elles habitaient avant l'accident, une personne est allée vivre chez son frère et deux ont été placées en centre d'hébergement (après une période d'essai à la maison de 6 à 12 mois). La décision finale correspond à la volonté de l'usager ou du proche aidant interrogé sept fois sur huit. Elle va à l'encontre de l'opinion des intervenants et/ou des enfants du couple quatre fois sur huit. C'est dire que les personnes cherchent à garder le contrôle sur leur destinée, autant que faire se peut.

Paul Ricœur (2001) définissait l'autonomie par une triple capacité : celle de dire (prendre la parole; assumer la position de sujet de ses actes et de ses mots), la capacité de se raconter (récapituler son histoire et entrevoir l'avenir afin de pouvoir imaginer sa vie autrement, envisager d'autres possibilités) et la capacité de faire (avoir une certaine influence sur les évènements, sur le cours des choses). On a vu que les personnes âgées et les proches aidants prennent la parole, formulent des préférences, expriment une volonté, assument une décision, et souvent avec vigueur; elles se racontent, font retour sur leur situations, sur la décision qu'elles ont prises, sur ce qu'elles ont l'intention de faire, les difficultés auxquelles elles font face et les choix qu'elles devront faire; enfin elles prennent de nombreuses initiatives, elles agissent, tentent de surmonter des obstacles, de trouver des solutions. Elles n'ont certes pas le contrôle sur tout, mais tentent de conserver ou d'accroître la prise qu'elles ont sur leur destin ${ }^{6}$.

\footnotetext{
${ }^{6}$ Comme l'avait bien vu Ricœur (2001) l'autonomie n'est pensable qu'en regard de la dépendance, l'action n'a de sens qu'en regard d'une contrainte ou d'une limite ; une capacité n'est pensable qu'en regard de ce qui lui résiste, lui fait obstacle; se dire capable ou autonome, c'est entendre surmonter cet obstacle, vaincre cette résistance.
} 


\section{Conditions de l'autonomie et expérience de la dépendance}

Les personnes âgées ayant subi un traumatisme craniocérébral interrogées, souffrent de divers handicaps et limitations (problème d'équilibre, paralysie partielle, perte de mémoire, incontinence, etc.), auxquels s'ajoutent d'autres ennuis de santé (diabète, problèmes cardiaques, basse pression, troubles de la vision); leur maintien à domicile nécessite des mesures de soutien souvent importantes, et une surveillance parfois continue. «J'ai besoin de surveillance, ça c'est sûr » (U2). Quant aux proches aidantes, en majorité des conjointes, elles sont elles-mêmes âgées et ont également des problèmes de santé importants : deux conjointes ont subi une opération depuis le retour à domicile de leur mari, elles sont épuisées, et la troisième ressent une grande fatigue. "Les enfants me disaient : "Maman, tu ne passeras pas à travers" » (P2). Leur propre capacité à s'occuper de leur proche et l'aide dont elles ont elles-mêmes besoin sont des préoccupations constantes. Si les intervenants semblent se préoccuper avant tout de sécurité, et les usagers et leurs proches de l'autonomie, la préoccupation pour la sécurité est également présente chez ces derniers, et parfois même fortement exprimée : «Qu'il tombe, je me disais : "Sacrifice! je ne peux pas toujours être à côté de lui" » (P3).

Les usagers et leurs proches n'ont pas de difficulté à formuler un désir et une volonté, à concevoir un projet et souvent à le mettre en œuvre. Mais ils ne trouvent pas en eux-mêmes tous les moyens pour le réaliser. Leur autonomie repose sur des appuis, des supports ou des ressources qui leur donnent les moyens de réaliser leur projet, de maintenir, restaurer ou développer leurs capacités (Gaulejac, 2009). Ces appuis, c'est d'abord le soutien des proches (conjoints, enfants et fratrie), par les services qu'ils rendent et la surveillance qu'ils exercent, ce sont aussi les ressources matérielles (achat de services, aménagement physique, résidence avec services), et les services professionnels offerts par les services publics, permettant d'accroître leurs capacités ou de compenser des limitations. On ne peut retourner chez soi, par exemple, que dans la mesure où un proche aidant peut fournir un soutien, exercer une surveillance, réaliser une part importante des tâches de la vie quotidienne (courses, repas, ménage et entretien, gestion des finances, aide pour le bain et les soins d'hygiène). L'autonomie d'une personne est fonction de son environnement social, ses limitations physiques pouvant ou non être compensées par cet environnement. Elle est fonction de l'étendue et de la qualité de son réseau d'aide; plus ce dernier est large et diversifié (conjoint, enfants, services publics et privés...), plus il lui est possible de continuer à faire des choses qu'elle aime ou à demeurer là où elle le souhaite. L'autonomie n'est pas la capacité de se passer des autres, mais au contraire la possibilité de prendre appui sur les autres. II y a là une sorte de paradoxe ou de cercle : l'autonomie repose sur des liens de dépendance, elle est dans un rapport circulaire avec la dépendance. Le sujet autonome n'advient pas dans le vide, uniquement en s'opposant aux autres, aux limites, aux contraintes, mais en prenant aussi appui sur les autres et sur l'environnement. Les personnes âgées et leurs proches en sont conscients, ils en ont au moins l'intuition : «Si on n'avait pas le CLSC, je ne pourrais être ici » (U2); "Si vous aviez dû rester seul, ça aurait pu être autre chose? - Exactement. Ça aurait changé les affaires »(U3).

La meilleure façon de répondre aux besoins de sa mère, dira une proche aidante, « c'était de la laisser faire ce qu'elle voulait faire. C'était de la laisser aller. Je me disais que, de toute façon, je suis là s'il arrive quelque chose " (P4). C'est dire que son autonomie repose largement sur la sécurité que procure la présence de sa fille. Outre l'aide essentielle des proches aidants - conjoints ou enfants -, les personnes reçoivent souvent l'aide des services publics et parfois de services privés: aide d'une auxiliaire de santé et de services sociaux pour prendre son bain, conseils d'une ergothérapeute pour réaménager l'espace ou effectuer des déplacements sécuritaires, informations du travailleur social 
touchant les services accessibles, les résidences, les activités adaptées à la condition de la personne, livraison à domicile de repas (popote roulante), livraison des médicaments par la pharmacie, etc. $A$ contrario, perdre tout soutien, c'est perdre toute autonomie.

On a vu plus haut que très souvent les personnes commencent par refuser l'aide, particulièrement celle des services publics. Mais très vite, elles comprennent qu'elles en auront besoin pour continuer à demeurer à domicile ou s'occuper de leur proche, sans qu'on sache toujours où trouver de l'aide, à qui s'adresser, ou quels sont les services existants. " J'étais tellement habituée de faire mes choses toute seule qu'il me semblait que ça n'avait pas de bon sens de demander quelqu'un. [...] Je m'arrange toute seule » $(P 1)$.

«À un moment donné, [la travailleuse sociale] m'a appelé. Elle m'a proposé d'avoir quelqu'un à la maison. Puis je n'ai pas voulu. On dirait que j'étais tannée... Puis un an après, je me suis dit : "Que j'ai donc mal fait. II me semble que j'aurais besoin d'un support. »

- Au début, on n'en sent pas le besoin?

- Non, pantoute. Quand c'est des choses que tu n'as pas vécues, tu ne peux pas savoir comment tu vas les vivre. Puis, ça dépend toujours de la façon dont tu es disposée aussi.

- La forme que vous avez, l'énergie?

- L’énergie que tu as. Oui, c'est ça exactement. Et [la travailleuse sociale], ça été mon sauveur » (P3).

Le manque d'aide et de soutien peut prendre une tournure dramatique, surtout pour les proches. «Ce n'est pas moi la psychologue, ce n'est pas moi la TS, s'exclame une proche aidante. Moi, j'en fais assez de même. Donne-moi un coup de main, fais quelque chose ! Je ne sais pas... » (P4). À propos de sa femme, un homme nous raconte : «Elle a été opérée, comme je vous l'ai dit. Elle n'était plus capable, c'était trop fatiguant pour elle. Elle est devenue malade à un moment donné »(U2). II leur a fallu de l'aide du CLSC pour tenir le coup. «À un moment donné, tu craques... », nous dit une proche aidante (P2). « Je faisais tout... nous confie une autre. Il était rendu avec 26 pilules. Là, je me suis dit : "Je vais paniquer !" » (P3). Une personne consent finalement à aller en centre d'hébergement lorsque sa femme doit être opérée au cœur. « II a dit : "Tu commences à vieillir, ça va être plus difficile pour toi. [...] Ici [au centre d'hébergement], j'ai des bons soins, ils s'occupent bien de moi” » (P1).

L'autonomie repose sur l'aide, il faut en quelque sorte consentir à dépendre des autres pour préserver un dernier espace de liberté et d'intimité. Mais il est des formes de soutien qui ne permettent plus d'exploiter ses capacités, rendent passives, placent dans une situation humiliante ou encore font que l'on se sent mis à l'écart, inutile et sans valeur aux yeux des autres. La dépendance est alors très mal vécue. « Je n'ai plus de char. [...] Je ne peux plus aller à la pêche, je ne peux plus rien faire », nous confie un usager (U5); « Je peux-tu sortir quand je veux et faire ce que je veux? » dit une autre avec impatience (U4); « II était cuisinier, mais après il n'a plus été capable... » constate une proche aidante. «Il aurait fallu qu'il ait des couches, puis là, il ne voulait plus en mettre » (P1). À propos de l'aide qu'il reçoit pour les soins d'hygiène, elle ajoute : “C'est dur d'avoir des étrangers. [...] C'est pas mal personnel » (P1). Un homme qui ne pourra rester avec sa fille nous confie son désarroi : « Moi, je n'ai plus de place...» (U5). II est privé d'un espace à soi, d'une place dans le monde.

Lorsqu'on lui a dit qu'il ne pourrait retourner chez lui, la réaction d'un usager fut : « Je leur ai dit au centre : "Ce que vous m'annoncez là, vous me coupez les jambes ben raide." Moi je ne suis plus capable 
de rien faire là » (U1). À l'idée d'être placé en centre d'hébergement, un autre usager réagit également très mal : " Il était tellement découragé, il pleurait tellement... II disait : "Je ne veux pas y aller, je veux rester chez nous avec toi." [...] Ça me faisait de la peine de le laisser » (P1). Faire l'expérience de la dépendance, c'est non seulement faire l'expérience de ses limites, devoir renoncer à des activités auxquelles on tient, devoir sacrifier son intimité, mais c'est être confronté à la mort, à sa propre mort.

«Elle est tellement autonome, que c'est un gros deuil pour elle. Elle dit : "Ça ne me donne plus rien de vivre si je ne peux pas patiner. Je fais quoi? Je reste écrasée devant la télé?" [...] Elle dit : "Je suis aussi bien de mourir tant qu'à ne plus rien faire." Parce que, dans sa tête à elle, c'est : “J'attends." T'attends quoi? Elle dit : “J'attends. Faut que j'attende que la popote vienne, faut que j'attende que le pharmacien vienne me livrer mes médicaments..." » (P4).

Un usager est encore plus explicite : « Je ne voulais pas aller dans un mouroir. » En pleurs, il explique : "Si on dit à quelqu'un : "Monsieur ou madame, vous êtes mieux de penser où vous allez aller, il faut changer de maison", ça veut dire que vous êtes fini. Comprenez-vous? II ne faut pas dire cela. II ne faut pas parler de cela. II faut parler de réhabilitation »(U2). Et un peu plus loin, toujours à propos de l'obligation de quitter sa maison, il ajoute : «C'est comme une condamnation à mort cela. » La mort est clairement associée à la dépendance; la mort c'est l'immobilité. Perdre des capacités c'est commencer à mourir :

« Je pleure parce que je pleure sur l'abandon. Moi, je considère que je suis mort en partie. [...] Le corps, une partie du corps nous abandonne. La mort, c'est ça. Et ne pas bouger, c'est la mort. Le mouvement c'est la vie. Tout être vivant bouge. Les morts ne bougent pas. [...] Nous, quand une partie de notre corps ne bouge pas, on est partiellement mort. C'est... c'est une mort partielle. Une mort lente »(U2).

On comprend mieux maintenant pourquoi l'hébergement institutionnel est si rapidement rejeté par les usagers et leurs proches, et que seules deux options - le domicile et l'hébergement - sont évoquées lors des entretiens. Les centres d'hébergement sont clairement associés à la mort : « J'ai dit [à l'intervenant] : "Je n'envoie pas ma mère dans une place de même, ce n'est pas vrai." C'était trop vieux » (P4). Sur les années passées en hébergement, un usager dira sans ambages à ses proches : « Vous m'avez fait perdre trois ans de ma vie »(P2). En revanche faire des exercices de réadaptation, conserver un espoir, si minime soit-il, de recouvrer des capacités, c'est demeurer en vie : "J'avais confiance qu'il en reprenne tout le temps »(P3). Vouloir retourner chez soi, c'est refuser le déclin c'est repousser la mort, c'est tenter de préserver ce qui donne sens à sa vie, ce qui nous maintient en vie sa relation de couple, ses activités préférées, un espace familier et rassurant. Une proche aidante nous rapporte ainsi les propos de sa mère : «Je m'en retourne chez nous, qu'ils me laissent donc vivre. [...] Qu'ils me laissent vivre pour le temps que j'ai à vivre. Qu'ils arrêtent, qu'ils décrochent, je ne suis pas un enfant $\gg(P 4)$.

L'autonomie repose sur de l'aide, des supports, disions-nous. Mais il est des situations où les appuis et les supports ne permettent plus de développer ses capacités. Au contraire, ils deviennent si importants et si contrôlants, qu'ils rendent la personne dépendante, passive; ils l'immobilisent et rendent toute parole inutile. Ou encore ils sont associés à l'abandon par ses proches, à la solitude, au manque de considération par les autres, qu'ils enlèvent tout désir, tout intérêt pour la vie. Le soutien est positif, désirable même, lorsqu'elle permet à la personne de «persévérer dans son être », pour reprendre la 
formule de Spinoza, de préserver sa puissance d'agir, lorsqu'il lui accorde encore une voix, la possibilité de s'exprimer, de la reconnaissance pour ce que l'on est et ce que l'on vaut ${ }^{7}$. C'est ce que les usagers et les proches semblent trouver dans leurs relations. Le soutien est négatif, lorsqu'il est associé à la mort, lorsqu'il prive de toute forme d'expression et d'action, lorsqu'il n'offre plus d'avenir dans lequel se projeter. C'est la solitude pour l'un, l'immobilité pour l'autre, ne plus pouvoir vivre dans le risque pour un troisième. La dépendance est alors privation, elle devient même violente.

\section{Entendre et soutenir leurs projets : réflexions pour le travail social}

En raison de son caractère exploratoire, la présente étude ne permet pas de conclure avec certitude, encore moins d'évaluer les pratiques d'intervention ou d'en définir les grands paramètres. Elle met cependant en lumière la manière dont les personnes se projettent dans le temps et formulent des projets - reprennent certaines activités, prennent soin de leur proche. Les personnes ont une volonté, des désirs qui prennent la forme d'un projet et elles sont prêtes à faire beaucoup de choses pour le réaliser. À l'image d'une personne diminuée et dépendante, elles résistent de différentes façons : en prenant des décisions, des initiatives et même des risques, en insistant lors des entretiens sur tout ce qu'elles sont encore capables de faire par elles-mêmes. Elles sont prêtes à prendre le risque de tomber et de se blesser, ou de s'épuiser; elles feront des choix et prendront des initiatives, et s'y tiendront, envers et contre tous s'il le faut. Avoir un projet et tout faire pour le réaliser est une nécessité : c'est résister au déclin et à la mort, refuser une vie qui perd tout sens et tout intérêt.

Le temps est marqué par l'incertitude. On ne sait pas quelles capacités la personne parviendra à récupérer, combien de temps l'aidant pourra assurer son soutien, sur quelles ressources elle pourra compter. Les besoins se transforment, la demande d'aide prend parfois un certain temps à émerger, les difficultés ou les progrès apparaissent après un certain temps. L'avenir demeure imprévisible, même si on peut anticiper des difficultés ou des solutions. Cette incertitude peut être perçue et analysée comme un risque, un argument pour décourager le retour à domicile; elle peut aussi être comprise comme une ouverture, une possibilité qui justifie de tenter quelque chose, de faire un essai.

Les témoignages des personnes invitent à réinterroger la pratique, à la revoir afin de se centrer sur les désirs des usagers, en prenant en compte non seulement les incapacités des usagers et les risques d'épuisement pour les proches, mais aussi leur volonté et leurs actions, les compromis et les difficultés qu'elles sont prêtes à assumer, même les risques qu'elles sont prêtes à courir. II faut entendre et comprendre leurs projets, qu'ils chercheront à mettre en œuvre, avec ou sans aide. Peut-être les intervenants doivent-ils changer de perspective. Ils ne sont pas uniquement les gardiens de la sécurité des personnes. Ils ont peut-être d'abord pour mission de soutenir les personnes dans ce qui leur paraît essentiel pour que leur vie continue d'avoir un sens, un intérêt, une valeur; de reconnaître leurs forces et leurs ressources; de chercher comment on peut rendre leur projet possible et viable; de comprendre jusqu'où les personnes sont prêtes à aller et les soutenir dans leurs essais. Si les intervenants conservent l'obligation de bien les informer sur les risques et les difficultés, les différentes options et les

\footnotetext{
${ }^{7}$ Persévérer dans son être c'est persévérer dans son essence : ce que l'on est, ce que l'on veut demeurer ou ce que l'on aspire à devenir. Ce n'est pas un état, mais un acte, une puissance d'agir. Cela ne veut pas dire qu'il n'y a pas de contrainte ou d'obligation, encore moins que l'on soit assuré du succès. Avoir une éthique, c'est précisément ne pas se laisser entièrement diriger par ces contraintes et ces obligations. Redisons-le, notre puissance d'agir ne se manifeste que dans la résistance.
} 
limites de l'aide qu'elles pourront recevoir, ils doivent aussi aider les personnes à rendre possible leur projet.

Dans cette perspective, la réadaptation ne vise pas uniquement à réparer des corps ou à compenser des déficits, mais également à soutenir la personne dans ses efforts pour conserver une autonomie (capacité de faire et d'entreprendre, de juger et de décider) et, plus largement, pour donner un sens à son expérience, c'est-à-dire pouvoir continuer à se projeter dans l'avenir, en fonction de ce qu'elle fut et de ce qu'elle entend demeurer, malgré les bouleversements.

GAGNON, Éric

VOYER, Manon

TERREAU, Paule

LACROIX, Nadine

FOURNIER ST-AMAND, Geneviève

OUELLET, Marie-Christine

\section{RÉFÉRENCES}

AQUESSS (Association québécoise d'établissements de santé et de services sociaux) (2011). Six cibles pour faire face au vieillissement de la population. Comité de travail de l'AQUESS, $52 \mathrm{p}$.

Atwal, A., A. Mclntyre et C. Wiggett (2012). " Risks with older adults in acute care settings: UK occupational therapists' and physiotherapists' perceptions of risks associated with discharge and professional practice ", Scandinavian Journal of caring sciences, vol. 26, n 2, p. 381-393.

Belleau, H., et C. Saint-Laurent (2005). "La décision d'hébergement des personnes âgées en institution : le point de vue des différents acteurs ", dans F.-R Ouellette, R. Joyal et R. Hurtubise, Familles en mouvance: Quels enjeux éthiques?, Québec, Presses de l'Université Laval, p. 317-332.

Benveniste, É. (1974). Problèmes de linguistique générale, tome 2, Paris, Gallimard.

Brown, S.B., A. Colantonio et H. Kim (2012). « Gender differences in discharge destination among older adults following traumatic brain injury », Health Care for Women International, vol. 33, n 10, p. 896904.

Degeneffe, C.E. (2001). « Family caregiving and traumatic brain injury », Health \& Social Work, vol. 26, $n^{\circ} 4$, p. 257-268. 
Ducharme, F. (2006). Famille et soins aux personnes âgées. Enjeux, défis et stratégies. Montréal, Beauchemin.

Flanagan, S.R., M.R. Hibbard et W.A. Gordon (2005). " The impact of age on traumatic brain injury », Physical Medecine and Rehabilitation Clinics of North America, vol. 16, n 1, p. 163-177.

Gagnon, É. (2015). «Avez-vous dit : autonomie? », Pratiques. Les cahiers de la médecine utopique, $\mathrm{n}^{\circ}$ 68 , p. $12-14$.

Gaulejac, V. (2009). Qui est «je »? Sociologie clinique du sujet, Paris, Seuil.

Giddens, A. (1991). Modernity and Self-ldentity: Self and Society in the Late Modern Age, Stanford (CA), Stanford University Press.

Goleburn, C.R., et C.J. Golden (2001). «Traumatic brain injury outcome in older adults: a critical review of the litterature », Journal of Clinical Geropsychology, vol. 7, n 3, p. 161-187.

LeBlanc, J., E. de Guise, N. Gosselin et M. Feyz (2006). « Comparison of functional outcome following acute care in young, middle-aged and elderly patients with traumatic brain injury », Brain Injury, vol. $20, n^{\circ} 8$, p. $779-790$.

Levine, J.M., et S.R. Flanagan (2012). « Traumatic brain injury in the elderly », dans N. Zasler, D. Katz et R. Zafonte (dir.), Brain Injury Medicine: Principles and Practice (Second Edition). New York, Demos.

Mosenthal, A., D. Livingston, R. Lavery, M.M. Knudson, S. Lee, D. Morabito, G.T. Manley, A. Nathens, G. Jurkovich, D.B. Hoyt et R. Coimbra, (2004). " The effect of age on functional outcome in mild traumatic brain injury: 6-month report of a prospective multicenter trial », The Journal of Trauma: Injury, Infection and Critical Care, vol. 56, n 5 , p. 1042-1048.

Ricœur, P. (2001). Le juste, tome 2, Paris, Éditions Esprit.

Sinnakaruppan, I., et D.M. Williams (2001). « Family carers and the adult head-injured: A critical review of carers' needs », Brain Injury, vol. 15, n 8, p. 653-672.

Teasell, R., S. Marshall, N. Cullen, M. Bayley, L. Rees et al. (2011). Evidence-Based Review of Moderate To Severe Acquired Brain Injury. Executive summary [En ligne]. Consulté le 19 ocotbre 2016. < http://www.abiebr.com/pdf/executivesummary.pdf

Testa, J.A., J.F. Malec, A.M. Moessner et A.W. Brown (2005). " Outcome after traumatic brain injury:effect of aging on recovery », Archives of Physical Medicine and Rehabilitation, vol. 86, $\mathrm{n}^{\circ}$ 9, p. 1815-1823. 